\title{
Internetgestützte Therapieprogramme: Gefahr für die traditionelle Psychotherapie?
}

\author{
Fritz Hohagen \\ Klinik für Psychiatrie und Psychotherapie, Universitätsklinikum Schleswig-Holstein, Campus Lübeck, Deutschland
}

Internet und Computer sind zum festen Bestandteil der Alltagswelt vieler Menschen geworden. Nie ging es einfacher und schneller, Informationen auszutauschen, Kontakte anzubahnen und mit Freunden, Experten oder Datenbanken zu kommunizieren. Via E-Mail, Chat-Programmen, Internet-Foren oder Web-Blogs lassen sich sowohl die Bewohner unterschiedlicher Kontinente als auch die nur zwei Zimmer voneinander entfernt sitzenden Kollegen zu virtuellen Gesprächspartnern machen. Was liegt da näher, als diese Kommunikationsformen auch für die Gestaltung psychotherapeutischer Kontakte zu nutzen?

In der psychosozialen Versorgung der sogenannten Industrieländer gewinnen Elemente computergestützter Therapieprogramme tatsächlich zunehmend an Bedeutung. Sie beziehen sich auf die ganze Bandbreite präventiver, diagnostischer, therapeutischer und rehabilitativer Angebote und Interventionen und können in vielfältiger Weise eingesetzt werden. Sie reichen von im Netz frei zugänglichen und anonym zu nutzenden Selbsttests zum Alkoholkonsum oder ScreeningFragebögen zur Depression über die EDV-basierte Auswertung von Patientenfragebögen im Behandlungsverlauf und deren unmittelbare Rückmeldung an Patient und Therapeut mittels Real-Time-Monitoring [Tominschek et al., 2008], das Computerspiel zur Unterstützung kognitiv-verhaltenstherapeutischer Behandlung von Kindern [Brezinka, 2007] bis hin zur Psychotherapie via E-Mail oder Audio-/Video-Chat, um nur einige Beispiele zu nennen.

Auch die Psychotherapieforschung beschäftigt sich bereits seit Längerem mit internetbasierter Psychotherapie. In den letzten 10-15 Jahren sind, vor allem in Schweden, den Niederlanden, der Schweiz, Deutschland, Großbritannien und Australien, für verschiedene Störungsbilder internetbasierte Therapieangebote entwickelt worden. Dabei haben besonders diejenigen Angebote, die auf den Prinzipien der kognitiven Verhaltenstherapie beruhen, stark zugenommen. Es gibt mitt- lerweile differenzierte und gut evaluierte Programme, die für den Transfer in die Regelversorgung potenziell geeignet sind. So konnte in randomisierten Studien gezeigt werden, dass die neuen Therapieansätze bei Angststörungen ebenso wirksam sind wie bei Depressionen leichten bis mittleren Grades [Barak et al., 2008; Spek et al., 2007]. Dennoch kommt die Implementierung dieser innovativen Behandlungspfade nur relativ langsam voran - vergleicht man sie mit der Vielzahl anderer Internet-Anwendungen, die uns den Alltag im beruflich-geschäftlichen wie privaten Leben mittlerweile erheblich erleichtern.

Welche Gründe spielen hierfür eine Rolle? Da sind zum einen berufsrechtliche Barrieren und die Restriktionen eines relativ starren Gesundheitssystems zu nennen, das die Behandlung eines Patienten nur mithilfe des Internets im Rahmen der gesetzlichen oder privaten Krankenversicherung nicht vorsieht. Zum anderen gibt es Vorbehalte sowohl seitens der Nutzer (User) als auch der herkömmlich im Face-to-FaceKontakt arbeitenden Psychotherapeuten, die besonders dann stark ausgeprägt sein dürften, wenn die therapeutische Beziehung als therapeutisches Agens betont wird. Auch die Frage nach der richtigen Indikationsstellung (Ist dieser Patient für diese Methode tatsächlich geeignet?) ist ohne persönlichen Kontakt zwischen Therapeut und Patient im Einzelfall nur schwer zu beantworten.

Eine kognitiv-verhaltenstherapeutische Behandlung via Internet im Selbsthilfe-Ansatz, die durch gelegentliche und auf das nötigste reduzierte Therapeutenkontakte per E-Mail begleitet wird, scheint jedoch ein kosteneffektives Vorgehen darzustellen, um mehr Patienten als bisher eine wirksame psychotherapeutische Behandlung zukommen zu lassen. Dies hat auch ökonomische Relevanz, was in den Niederlanden im Fall von «Interapy», einem Programm zur Behandlung von posttraumatischen Belastungsstörungen, dazu geführt hat, dass diese ausschließlich online durchgeführte Psychotherapie von

\section{KARGER}

Fax +497614520714

Information@Karger.de

www.karger.com (c) 2009 S. Karger GmbH, Freiburg

Accessible online at:

www.karger.com/ver 
den Krankenkassen anerkannt und bezahlt wird [Lange et al., 2003] - eine Situation, die in Deutschland noch undenkbar ist, da eine reine Fernbehandlung hierzulande außerhalb von wissenschaftlichen Modellprojekten nicht gestattet ist.

Neben dem ökonomischen Nutzen für den einzelnen Kostenträger und möglicherweise das Gesundheitssystem insgesamt, ist die relative Anonymität und Niedrigschwelligkeit eines solchen Angebots besonders für solche Patienten attraktiv, die sich einem herkömmlichen Therapiesetting z.B. aus Gründen der Scham oder aus praktischen Gründen im Zweifelsfall eher verweigern würden. Hierin liegt eine Chance, den Zugang zur Psychotherapie für neue Zielgruppen $\mathrm{zu}$ erschließen. $\mathrm{Zu}$ denken ist da z.B. an Patienten mit unregelmäßigen Arbeitszeiten oder in entlegenen ländlichen Gebieten, wo kein ausreichendes Therapeutenangebot besteht. Sie können sich unabhängig von Zeit und Raum mittels Internet in einen therapeutischen Kontakt und Dialog begeben. So erhöht internetbasierte Therapie die Flexibilisierung von Therapeut-Patient-Kontakten bei - je nach Programm - gleichzeitig potenziell standardisierter Vorgehensweise. Damit könnten internetbasierte Psychotherapieangebote, die evaluiert sind, bei denen der Datenschutz gewährleistet ist (verschlüsselte Übertragungswege) und die sich einer Qualitätskontrolle unterziehen, möglicherweise der Forderung Riefs [2008] nach kosteneffektiven, zeitlich kondensierten und hoch effizienten Behandlungen angesichts von Unterversorgung und durchschnittlichen Wartezeiten von 4-8 Monaten auf einen Psychotherapieplatz in angemessener Form Rechnung tragen.

Allerdings dürften diese neuen Medien in der Psychotherapie für bestimmte Patientengruppen nicht geeignet sein. $\mathrm{Zu}$ denken ist hier an schwerer gestörte Patienten, an alte oder sehbehinderte Patienten, wenn der Internetzugang und dessen Handhabung nicht barrierefrei erfolgen kann, oder an Personen, die sich die nötige technische Ausstattung (Hardund Software, Internetgebühren) nicht leisten können oder wollen. Außerdem sind Fragen z.B. der Differenzialindikation (Welche Gruppen profitieren von welchen Programmen?) und der therapeutischen Beziehungsgestaltung noch offen und bedürfen der weiteren Forschung. Die wenigen Studien, die der Frage der therapeutischen Beziehung nachgegangen sind, konnten zeigen, dass es in internetbasierten Psychotherapien durchaus möglich ist, eine gute therapeutische Beziehung aufzubauen, und dass die Qualität dieser Beziehung auch den Behandlungserfolg beeinflusst [Berger und Andersson, im Druck].

Als Antwort auf die übergeordnete Frage kann meines Erachtens gelten: Internetbasierte Psychotherapien gefährden die traditionellen Formen psychotherapeutischen Arbeitens nicht. Sie stellen keine Konkurrenz dar, sondern können - ähnlich wie z.B. Telefonkontakte - eine sinnvolle Bereicherung des herkömmlichen Psychotherapiesettings sein. In ihrer ausschließlich internetbasierten Vollversion ermöglichen sie darüber hinaus bislang unerreichbaren Patientengruppen erstmals Zugang zu Psychotherapie bzw. weniger schwer gestörten Patienten eine praktische Alternative zum traditionellen Psychotherapiesetting. Internetbasierte Psychotherapieformen müssen sich - unterstützt durch intensive Begleitforschung - ihren Stellenwert innerhalb der Psychotherapielandschaft jedoch erst noch erarbeiten. Gerade die Verhaltenstherapie ist aufgefordert, sich an der Weiterentwicklung dieses Trends beim Einsatz neuer Medien, von denen sie besonders stark profitieren dürfte, zu beteiligen.

\section{Literatur}

Barak A, Hen L, Boniel-Nissim M, Shapira N: A comprehensive review and a meta-analysis of the effectiveness of internet-based psychotherapeutic interventions. J Technol Human Serv 2008;26(2/4):109_ 160.

Berger T, Andersson G: Internetbasierte Psychotherapien: Besonderheiten und empirische Evidenz. Psychother Psychosom Med Psychol 2009, in press.
Brezinka V: Schatzsuche - ein Computerspiel zur Unterstützung der kognitiv-verhaltenstherapeutischen Behandlung von Kindern. Verhaltenstherapie 2007;17:191-194.

Lange A, van den Ven J-P, Schrieken B: Interapy: Treatment of post-traumatic stress through the Internet. Cogn Behav Ther 2003;32:110-124.

Rief W: 450 Millionen Euro Sondermittel für Verhaltenstherapie - ist die britische Initiative für psychisch Kranke ein Modell für Deutschland? Verhaltenstherapie 2008;18:212-213.
Spek V, Cuijpers P, Nyklícek I, Riper H, Keyzer J, Pop V: Internet-based cognitive behaviour therapy for symptoms of depression and anxiety: A metaanalysis. Psychol Med 2007;37:319-328.

Tominschek I, Schiepek G, Mehl C, Maier K, Heinzel S, Bauhofer C, Berbic B, Zaudig M: Real-TimeMonitoring in der Behandlung von Zwangsstörungen: Technologie und Fallbeispiel. Verhaltenstherapie 2008;18:146-152. 